\title{
OTIOTOMICS
}

Revista de economía, empresa y sociedad

Dossier «Realidades y desafíos de la Unión Europea»

COMPROMISOS Y REALIDADES

\section{Políticas de I+D y convergencia productiva en la Unión Furopea}

\section{Albert Puig Gómez}

Profesor de los Estudios de Economía y Empresa (UOC)

RESUMEN La sostenibilidad de la Unión Europea pasa por la disminución de sus divergencias estructurales. Bajo esta premisa, y teniendo en cuenta que la política de I+D es clave para avanzar en la transformación productiva de los países de la UE menos avanzados tecnológicamente, la Comisión Europea acordó con cada uno de los Estados miembros unos compromisos relativos al esfuerzo en I+D (gasto en I+D en relación con el producto interior bruto) que deberían alcanzar en el año 2020. En nuestro trabajo ponemos de manifiesto la baja probabilidad de alcanzar esos compromisos, especialmente para los países con mayores necesidades de transformación productiva, entre ellos España. Y en ese mal pronóstico desempeñan un papel clave las políticas de austeridad impuestas por la propia Comisión Europea a esos mismos países, las cuales han golpeado de manera dramática en sus presupuestos públicos de I+D. En este escenario, la UE está avanzando hacia una mayor divergencia, lo cual genera mayores dudas acerca de su propia viabilidad.

PALABRAS CLAVE convergencia productiva; investigación y desarrollo (I+D); crisis económica; políticas de austeridad

\section{$R \& D$ policies and productivity convergence in the European Union}

\begin{abstract}
The sustainability of the European Union depends upon reducing its structural divergences. Under this premise, and taking into account that R\&D policies are key to transforming productivity in EU countries that are technologically less advanced, the European Commission obtained from each of its member states commitments regarding the efforts towards $R \& D$ (expenditure as a percentage of GDP) that they would be making by the year 2020. In this study we demonstrate the unlikelihood of these commitments being fulfilled, especially in those countries with a greater need for productive transformation, of which Spain is one example. This predicted failure is heavily influenced by the austerity measures imposed on the aforementioned countries by the very same European Commission, which resulted in major cuts in their $R \& D$ expenditure. In this context, the EU is moving towards greater degrees of divergence, generating serious doubts about its continued feasibility.
\end{abstract}

KEYWORDS productivity convergence; Research and Development (R\&D); economic crisis; austerity policies 


\section{Introducción}

La profunda crisis que azota a la Unión Europea (UE) tiene uno de sus orígenes en las divergencias productivas existentes entre sus Estados miembros. Esta evidencia, que ya fue puesta de manifiesto por diversos analistas mucho antes de la llegada de la actual crisis, se «oficializó» en 2008, haciendo emerger la necesidad de afrontar un intenso proceso de transformación productiva en los países tecnológicamente menos avanzados, que les permitiera una inserción más sólida y sostenible tanto en el mercado de la Unión Europea como en el mercado global.

En este sentido, existe un amplio acuerdo en que una de las principales líneas de actuación para avanzar en el deseado cambio productivo debe pasar por la potenciación de las actividades de investigación y desarrollo (I+D). Para ello, la Comisión Europea acordó con cada uno de los Estados miembros de la UE unos compromisos relativos al esfuerzo en I+D (gasto en I+D en relación con el producto interior bruto, PIB) que cada uno debería alcanzar en el año 2020.

Nuestro trabajo analiza, en primer lugar, la evolución del esfuerzo en I+D en los países más avanzados de la Unión Europea (UE-15), tanto en el periodo de crisis como en el periodo previo de expansión económica; posteriormente, y a partir de los datos del pasado, presentamos los escenarios que se identifican en relación con el objetivo de lograr los compromisos «acordados» con la Comisión Europea en términos de esfuerzo en I+D; en tercer lugar, en función de los datos sobre el origen de los fondos destinados a l+D en cada uno de los países de la UE-15, y centrándonos en el caso español, analizamos el impacto de la crisis y de las políticas de austeridad impuestas por la troika en los presupuestos públicos de I+D de los países europeos tecnológicamente menos avanzados y, en consecuencia, más necesitados de avanzar en el sentido deseado de cambio de modelo productivo ${ }^{1}$. Cerramos el trabajo con el apartado de conclusiones.

\section{El gasto en I+D en los países de la Unión Europea}

En la tabla 1 se presentan los datos promedio de esfuerzo en I+D llevados a cabo por los países de la UE-15 (Estados miembros de la UE ya antes de la ampliación de 2004) en dos periodos distintos: los años de mayor expansión económica (2002-2007) y en seis años de crisis económica (2008-2013). Cabe señalar que el concepto «esfuerzo en I+D» incluye tanto los gastos en I+D llevados a cabo por el sector público como los realizados por parte del sector empresarial privado.

Tabla 1. Esfuerzo promedio en I+D (en porcentaje del PIB)

\begin{tabular}{|l|c|c|c|c|}
\hline & \multicolumn{2}{|c|}{ 2002-2007 } & \multicolumn{2}{c|}{ 2008-2013 } \\
\hline & & Rank & & Rank \\
\hline Suecia & 3,43 & 1 & 3,31 & 2 \\
\hline Finlandia & 3,32 & 2 & 3,57 & 1 \\
\hline Dinamarca & 2,45 & 3 & 2,97 & 3 \\
\hline
\end{tabular}

1. El término troika hace referencia a la actuación conjunta del Banco Central Europeo, la Comisión Europea y el Fondo Monetario Internacional en los rescates financieros (de deuda pública o bancarios) de algunos países de la Unión Europea. A cambio de obedecer a la troika, el país que lo necesite recibirá financiación del FMl o del BCE. De esta manera, el país financiado por la troika se encuentra intervenido dado que al seguir sus directrices pierde gran parte de su independencia política. La troika realiza la supervisión de la aplicación de los llamados programas de consolidación fiscal. Si el país deja de obedecer a la troika, dejará de obtener financiación. 


\begin{tabular}{|c|c|c|c|c|}
\hline & \multicolumn{2}{|c|}{$2002-2007$} & \multicolumn{2}{|c|}{$2008-2013$} \\
\hline & & Rank & & Rank \\
\hline Alemania & 2,44 & 4 & 2,78 & 4 \\
\hline Austria & 2,27 & 5 & 2,71 & 5 \\
\hline Francia & 2,08 & 6 & 2,18 & 6 \\
\hline EU-15 & 1,84 & & 2,03 & \\
\hline Bélgica & 1,83 & 7 & 2,10 & 7 \\
\hline Países Bajos & 1,78 & 8 & 1,82 & 8 \\
\hline Reino Unido & 1,66 & 9 & 1,68 & 9 \\
\hline Luxemburgo & 1,64 & 10 & 1,43 & 11 \\
\hline Irlanda & 1,17 & 11 & 1,55 & 10 \\
\hline España & 1,09 & 12 & 1,31 & 13 \\
\hline Italia & 1,08 & 13 & 1,22 & 14 \\
\hline Portugal & 0,83 & 14 & 1,46 & 12 \\
\hline Grecia & 0,56 & 15 & 0,67 & 15 \\
\hline
\end{tabular}

Fuente: Cálculos propios a partir de Eurostat.

De la tabla se desprende que son los países con una especialización productiva basada en productos de alta y media-alta tecnología ${ }^{2}$ los países que realizan constantemente (tanto en expansión como en crisis económica) un mayor esfuerzo en I+D.

Esta constatación pone de manifiesto que la política de I+D de la UE, tal y como está planteada, no contribuye a avanzar hacia una mayor convergencia productiva, sino que, por el contrario, los países menos avanzados del grupo (España, Grecia, Irlanda, Italia y Portugal) retroceden y divergen cada vez más del grupo de países más avanzados. Es evidente que si partiendo de una situación de desequilibrio, el que va delante invierte más que el que va detrás precisamente en el componente del gasto que permite avanzar en la escala de contenidos tecnológicos de sus producciones, el objetivo de acercamiento y de convergencia se aleja ${ }^{3}$.

Con el propósito de hacer frente a este hecho, la Comisión Europea estableció como uno de los cinco objetivos prioritarios del programa Europa 2020, puesto en marcha en el año 2010, alcanzar un esfuerzo en I+D del $3 \%$ del PIB de la UE (en este caso UE-28) para el año $2020^{4}$. Para alcanzar esta cifra, y considerando los puntos de partida de cada uno de los países, se estableció de manera consensuada sus respectivos targets.

2. La clasificación de las producciones según sus intensidades tecnológicas se puede consultar en la página web de la OCDE (http:// www.oecd.org/sti/ind).

3. Puede sorprender el hecho de que los países menos avanzados de la UE-15 realicen un esfuerzo en l+D mayor en el periodo de crisis que en el anterior de expansión. Esto es así porque en todos los países, también en el conjunto de los menos avanzados, la tendencia desde mediados de la década de los noventa ha sido la de aumentar el esfuerzo realizado en l+D. Por ejemplo, en el caso de España, el dato para 1997 era del 0,78\%, mientras que en 2002 fue del 0,96 y en 2007 del 1,09\%, lo cual conduce a un promedio inferior al que tiene lugar para 2008-2013. El hecho negativo es que esa tendencia creciente es lo que se va a revertir a partir de 2011 con las políticas de austeridad.

4. Para conocer en qué consiste el programa Europa 2020, se puede consultar el enlace http://ec.europa.eu/europe2020/index_en.htm. 
En la tabla 2 se presentan estos objetivos-país, el promedio de crecimiento anual del esfuerzo en I+D llevado a cabo en el periodo 2000-2011, así como el requerido para alcanzar el objetivo 2020.

Tabla 2. Objetivo 2020

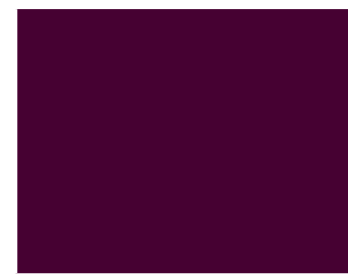

Finlandia

Suecia

Dinamarca

Alemania

Austria

Eslovenia

Estonia

Francia

Bélgica

Holanda

UE-28

Rep. Checa

Reino Unido

Irlanda

Portugal

Luxemburgo

España

Italia

Hungría

Lituania

Polonia

Croacia

Malta

Letonia

Eslovaquia

Grecia

Bulgaria

Rumanía

Chipre

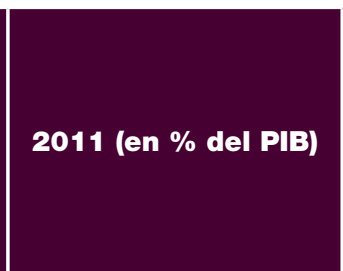

3,78

3,37

3,09

2,84

2,75

2,47

2,38

2,25

2,04

2,04

2,03

1,84

1,77

1,72

1,50

1,43

1,33

1,25

1,21

0,92

0,77

0,75

0,73

0,70

0,68

0,60

0,57

0,48

0,48

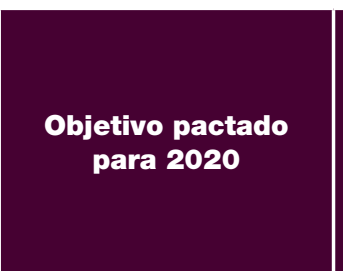

4,00

4,00

3,00

3,00

3,76

3,00

3,00

3,00

3,00

2,50

3,00

na

na

2,00

3,00

2,45

2,00

1,53

1,80

1,90

1,70

1,40

0,67

1,50

1,20

0,67

1,50

2,00

0,50
Promedio de crecimiento anual del esfuerzo en I+D durante 2000-2011
Promedio de crecimiento anual del esfuerzo en I+D requerido en el periodo 2011-2020 para alcanzar el objetivo 2020

Fuente: DG for Research and Innovation (2013). 
A partir de los datos de la tabla anterior, se identifican cinco grupos de países:

1) Aquellos que ya han llegado o están a punto de alcanzar su objetivo: Finlandia, Dinamarca, Alemania, Malta y Chipre. Este grupo incluye tanto países con altos esfuerzos en I+D (Finlandia, Dinamarca y Alemania) como otros con muy baja intensidad en I+D (Malta y Chipre). Todos ellos, sin embargo, situaron su objetivo en un nivel que está dentro de su alcance dado el contexto económico nacional.

2) Países que están en camino de llegar a su objetivo en función de su tasa promedio de progreso a lo largo del periodo 2000-2011: Austria, Estonia, Grecia, Hungría, Irlanda, Italia y Eslovenia. Este grupo incluye países con objetivos relativamente ambiciosos (Austria y Hungría), habida cuenta de su posición inicial, así como otros cuyos objetivos pueden ser vistos como más fáciles de alcanzar (Estonia, Grecia, Irlanda, Italia y Eslovenia).

3) Países que necesitan aumentar su tasa de esfuerzo en I+D para alcanzar su objetivo: Bélgica, Francia, Países Bajos, España y Suecia. Si bien estos países actualmente no están en camino de alcanzar su objetivo, el esfuerzo necesario (es decir, la diferencia entre la tasa de incremento requerida y su tendencia a largo plazo) es menor o comparable a la media de la UE .

4) Los que necesitan elevar sustancialmente su tasa de esfuerzo en I+D para alcanzar su objetivo y cuyos esfuerzos requeridos exceden de la media de la UE: Bulgaria, Letonia, Lituania, Luxemburgo, Polonia, Portugal, Rumanía y Eslovaquia. En este grupo, los países han establecido objetivos muy ambiciosos respecto tanto a los niveles de inicio como a sus tendencias del pasado. En consecuencia, las tasas de crecimiento requeridas son muy superiores a la media de la UE.

5) Finalmente, un pequeño grupo de países no tienen fijada una meta de esfuerzo en I+D: Reino Unido y la República Checa.

\section{La financiación de la I+D}

En la siguiente tabla se muestran los orígenes de los fondos dedicados a la I+D en los países de la UE-15 tanto en el periodo expansivo, que va de 1997 a 2006, como en el recesivo, iniciado en 2008.

Tabla 3. Gastos en I+D por sectores de origen de los fondos (en \% del total)

\begin{tabular}{|c|c|c|c|c|c|c|c|c|c|c|}
\hline & \multicolumn{2}{|c|}{$\begin{array}{c}\text { Sector } \\
\text { empresarial }\end{array}$} & \multicolumn{2}{|c|}{ AA. PP. } & \multicolumn{2}{|c|}{ Universidad } & \multicolumn{2}{|c|}{$\begin{array}{l}\text { Sector privado } \\
\text { sin ánimo de } \\
\text { lucro }\end{array}$} & \multicolumn{2}{|c|}{ Extranjero } \\
\hline & $97-06$ & $08-13$ & $97-06$ & $08-13$ & $97-06$ & $08-13$ & $97-06$ & $08-13$ & $97-06$ & $08-13$ \\
\hline Suecia & 66,98 & 58,20 & 24,28 & 27,50 & 0,30 & 0,75 & 2,88 & 0,93 & 5,60 & 10,75 \\
\hline Finlandia & 68,23 & 65,90 & 26,64 & 24,87 & 0,21 & 0,17 & 0,91 & 1,20 & 4,01 & 7,82 \\
\hline Alemania & 65,97 & 66,14 & 31,01 & 29,50 & sd & sd & 0,38 & 0,25 & 2,66 & 4,04 \\
\hline Dinamarca & 59,95 & 60,84 & 28,53 & 28,18 & sd & sd & 2,85 & 2,97 & 8,40 & 7,46 \\
\hline Francia & 52,46 & 53,40 & 38,10 & 36,96 & 0,92 & 1,18 & 0,89 & 0,72 & 7,61 & 7,56 \\
\hline Austria & 44,16 & 45,43 & 35,79 & 37,42 & 0,30 & 0,70 & 0,38 & 0,50 & 19,58 & 16,47 \\
\hline
\end{tabular}

5. En el caso español, además, cabe señalar que el objetivo inicialmente pactado fue del 3\%, que se rebajó al 2\% en el marco del Programa de Estabilidad «acordado» con la troika en 2011. 


\begin{tabular}{|c|c|c|c|c|c|c|c|c|c|c|}
\hline & \multicolumn{2}{|c|}{$\begin{array}{c}\text { Sector } \\
\text { empresarial }\end{array}$} & \multicolumn{2}{|c|}{ AA. PP. } & \multicolumn{2}{|c|}{ Universidad } & \multicolumn{2}{|c|}{$\begin{array}{c}\text { Sector privado } \\
\text { sin ánimo de } \\
\text { lucro }\end{array}$} & \multicolumn{2}{|c|}{ Extranjero } \\
\hline & $97-06$ & $08-13$ & $97-06$ & $08-13$ & $97-06$ & $08-13$ & $97-06$ & 08-13 & $97-06$ & $08-13$ \\
\hline Bélgica & 62,03 & 59,35 & 23,38 & 24,33 & 2,52 & 3,03 & 0,46 & 0,42 & 11,61 & 12,68 \\
\hline Países Bajos & 50,45 & 47,58 & 36,15 & 36,43 & 0,15 & 0,38 & 2,08 & 2,33 & 11,20 & 12,08 \\
\hline UE-15 & 55,55 & 51,03 & 34,18 & 34,60 & 0,68 & 1,27 & 1,57 & 1,42 & 8,22 & 10,81 \\
\hline Reino Unido & 45,22 & 45,32 & 30,78 & 30,30 & 1,03 & 1,18 & 4,59 & 4,82 & 18,34 & 18,35 \\
\hline Luxemburgo & 83,60 & 45,73 & 11,83 & 29,87 & sd & 0,08 & 0,10 & 0,48 & 4,50 & 15,50 \\
\hline Irlanda & 61,71 & 50,62 & 27,37 & 29,70 & 1,42 & 0,96 & 0,34 & 0,38 & 9,13 & 18,26 \\
\hline Italia & 40,05 & 44,84 & 48,85 & 42,02 & 0,75 & 1,06 & 2,25 & 2,47 & 8,15 & 9,14 \\
\hline España & 48,26 & 44,26 & 40,41 & 45,38 & 4,33 & 3,70 & 0,87 & 0,52 & 6,13 & 6,04 \\
\hline Portugal & 30,88 & 45,32 & 60,72 & 43,84 & 1,10 & 3,72 & 2,20 & 2,37 & 5,11 & 4,03 \\
\hline Grecia & 29,13 & 32,50 & 47,18 & 52,72 & 2,00 & 2,17 & 0,85 & 0,92 & 20,88 & 11,70 \\
\hline
\end{tabular}

Fuente: Eurostat.

Algunas de las principales observaciones de tendencia que se desprenden de la tabla anterior son las siguientes:

a) Que en los países que más gastan en I+D en relación con sus respectivos PIB (tabla 1) (Suecia, Finlandia, Alemania, Dinamarca, Francia) el principal agente financiador es el sector empresarial privado, mientras que en los países que menos gastan en I+D (Italia, España, Portugal y Grecia) el agente financiador más importante es el sector público.

b) Que en la mayor parte de los países la importancia relativa del sector empresarial privado en los años de crisis ha disminuido (y en los casos en que esto no ha sido así, el aumento de la importancia del sector empresarial es notablemente modesto).

Del análisis de cómo se ha compensado esta disminución relativa se identifican dos grupos de países. Por un lado, aquellos que combinan un aumento de la importancia relativa del sector público en la financiación de la I+D, consecuencia de aumentar los presupuestos públicos en estos conceptos, con un sensible aumento de la inversión extranjera en los mismos ámbitos (Suecia, Alemania, Dinamarca, Bélgica, Países Bajos, Irlanda). En estos países, por lo tanto, el gasto público en I+D aumenta pero este aumento es insuficiente para compensar la disminución de la inversión empresarial. El sector exterior se ocupa de cubrir la diferencia.

Por otro lado, en los países de nivel tecnológico menos avanzado dentro de la UE-15 y por lo tanto con mayores necesidades de transformación productiva (Italia, España, Portugal y Grecia), las caídas de la importancia relativa del sector empresarial en los gastos en I+D han sido compensadas exclusivamente con un aumento del peso del sector público. Pero este aumento relativo no es el resultado de aumentos en términos absolutos de gasto público en estos ámbitos, sino que tiene lugar a pesar de los importantes recortes presupuestarios que, tanto en términos generales como específicamente en el ámbito de la I+D, han venido sufriendo estos países desde la imposición por parte de la troika de las llamadas políticas de austeridad.

España constituye un ejemplo ilustrativo de este hecho ${ }^{6}$. El total de gastos contemplado en los Presupuestos Generales del Estado (PGE) aumentó hasta 2009, año en que fue todavía un 10,46\% mayor al de 2008. Los primeros PGE restrictivos fueron los del 2010, con un descenso global del 1,63\% en relación con el año anterior

6. Para un análisis más detallado del caso español, ver Puig, Soldevila, De la Cámara y Mañé (2014). 
(tabla 4). Estos presupuestos también significaron el primer año en el que la dotación para I+D disminuyó, y lo hizo en un porcentaje sensiblemente superior a la caída global (un 4,04\%), lo cual significó pasar del 2,90 al 2,83\% en términos de la importancia relativa que los gastos en I+D tuvieron dentro del presupuesto total.

Tabla 4. Variación presupuestaria en España

\begin{tabular}{|l|c|c|c|c|c|c|} 
& $\mathbf{\Delta 1 0 / 0 9}$ & $\mathbf{\Delta 1 1 / 1 0}$ & $\mathbf{\Delta 1 2 / 1 1}$ & $\mathbf{\Delta 1 3 / 1 2}$ & $\mathbf{\Delta 1 4 / 1 3}$ & $\mathbf{\Delta 1 5 / 1 4}$ \\
\hline Total PGE & $-1,63$ & $-11,89$ & $-1,94$ & 8,46 & 3,64 & 4 \\
\hline Despesa pública R+D & $-4,04$ & $-7,38$ & $-26,39$ & $-6,13$ & 3,6 & 4,24 \\
\hline
\end{tabular}

Fuente: Elaboración propia a partir de datos del informe de CC. OO. (2014), de los informes de la COSCE (2014 y 2015) y de los Presupuestos Generales del Estado.

Los presupuestos de 2011, ya completamente dentro del periodo de las políticas de austeridad, sufrieron un recorte total del 11,89\% con relación a 2010, mientras que la disminución que se aplicó a los gastos en I+D fue inferior al global $(7,18 \%)$; de esta manera, su peso dentro del conjunto de los PGE volvió a aumentar hasta el 2,97\% (el mayor en los últimos seis años).

Con la llegada al poder del Partido Popular en enero de 2012 los recortes presupuestarios se intensifican. En los primeros presupuestos aprobados, ya a mediados de 2012, la l+D se llevó una de las peores partes en el reparto de recortes, dado que, en el marco de una disminución global del 1,94\%, los gastos en esos ámbitos cayeron en un $26,39 \%$ en relación con los de 2011.

Por su parte, el presupuesto de gastos para 2013 aumentó en un 8,46\% con relación al de 2012, debido fundamentalmente a los intereses de la deuda pública (el presupuesto de gastos no financieros aumentó en un 2,2\%). Sin embargo, en este contexto de aumento del gasto público, el presupuesto de I+D para 2013 siguió disminuyendo, y lo hizo en un 6,13\% menos que el de 2012.

Finalmente, en los PGE para los años 2014 y 2015, la cantidad total presupuestada para I+D aumenta en porcentajes muy similares a los del aumento general de los gastos, manteniendo el volumen de gasto en I+D en alrededor del 1,5\% del total de gasto público previsto. Estas subidas, sin embargo, no pueden ser calificadas más que de tibias subidas, puesto que de hecho no compensan ni de lejos los fuertes recortes de los años anteriores.

En síntesis, el caso español constituye un ejemplo muy evidente de la incoherencia entre los discursos y los planes oficiales acerca de las políticas que implementar en el ámbito de la I+D y las medidas efectivamente llevadas a cabo. Y en esta incoherencia desempeñan un papel clave tanto la sumisión de los gobiernos de los países afectados, como las autoridades europeas, que acuerdan unos objetivos en términos de esfuerzo en I+D que luego acompañan con la imposición de unas políticas de austeridad que las hacen inalcanzables.

\section{Conclusiones}

Uno de los principales retos que afronta la Unión Europea en los próximos e inmediatos años es el de disminuir sus desequilibrios productivos y avanzar hacia una mayor convergencia estructural. El logro de este propósito debería conducir a mayores cotas de convergencia real (en niveles salariales, por ejemplo) y, entre otras cosas, a hacer más sencilla la gestión macroeconómica de la zona euro. Y, sin lugar a dudas, la política de I+D es clave para alcanzar ese objetivo.

Sin embargo, de los datos presentados y analizados a lo largo del presente texto, se desprende que, hasta hoy, el camino recorrido va en sentido inverso. Los países de la UE más avanzados tecnológicamente invierten en I+D más que los países tecnológicamente menos avanzados. Y esta es una constante que se identifica tanto en el periodo expansivo como en el de crisis. Es más, desde el inicio de la crisis, los países más avanzados de la UE15 han aumentado su esfuerzo en I+D en comparación al esfuerzo promedio (carácter anticíclico de la política de 
I+D) que realizaron a lo largo del periodo expansivo, mientras que los países menos avanzados lo han disminuido (carácter procíclico de la política de I+D).

Uno de los principales factores responsable de este hecho han sido las políticas de austeridad impuestas por la troika a los llamados países del sur de Europa, las cuales han incidido fuertemente en los gastos destinados a la I+D. Y es que estos países, aunque siempre lejos de los niveles de gasto en estos ámbitos llevados a cabo por los países europeos tecnológicamente más avanzados, habían ido progresivamente aumentando sus esfuerzos en I+D a lo largo de los últimos diez o quince años. Esta tendencia es la que se revierte a partir de 2011 como consecuencia de las medidas de austeridad impuestas por la propia Comisión Europea.

De ello se desprende que el objetivo de que los países tecnológicamente menos avanzados se acerquen productivamente a los más avanzados se aleja, lo cual amenaza a los primeros con consolidar un modelo productivo que los separe cada día más de los segundos. Pero solo con una financiación creciente y sostenida en el tiempo de las actividades de I+D (los resultados en este ámbito son claramente de signo acumulativo) es posible avanzar en la construcción de un sistema económico y productivo competitivo y sostenible.

\section{Referencias bibliográficas}

CC. OO. (2014). La industria en el Proyecto de PGE 2014. Federación de Industria de la Confederación Sindical de Comisiones Obreras. 23 páginas.

COSCE (2014). La inversión en I+D+i en los Presupuestos Generales del Estado aprobados para 2014. Confederación de Sociedades Científicas de España. 7 páginas.

COSCE (2014). Informe de urgencia sobre la inversión en I+D en el Proyecto de Presupuestos Generales del Estado para 2015. Confederación de Sociedades Científicas de España. 4 páginas.

EUROPEAN COMISSION (2013). Country Profile of Spain. DG for Research and Innovation. 26 páginas.

PUIG, A.; SOLDEVILA, V.; DE LA CÁMARA, C.; MAÑÉ, A. (2014). «La política de I+D+i en España en el período 2012-2014: incoherencia entre discurso y hechos». En: SÁNCHEZ, A.; CARPI, J. A. (dirs.). Crisis y Política Económica en España. Un análisis de la Política Económica actual. Thomson Reuters Aranzadi, págs. 229-248.

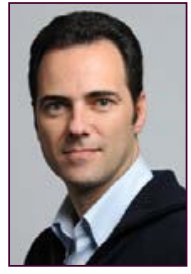

Albert Puig Gómez apuiggo@uoc.edu Profesor de los Estudios de Economía y Empresa (UOC)

Licenciado en Ciencias Económicas y doctor en Economía por la Universidad de Barcelona. Dirige el máster universitario de Análisis del entorno económico de la UOC.

Los textos publicados en esta revista están -si no se indica lo contrario- bajo una licencia Reconocimiento-Sin obras derivadas 3.0 España de Creative Commons. Puede copiarlos, distribuirlos y comunicarlos públicamente siempre que cite su autor y la revista y la institución que los publica (autoría, nombre de la revista, institución editora); no haga con ellos obras derivadas. La licencia completa se puede consultar en http://creativecommons.org/licenses/by-nd/3.0/es/deed.es.

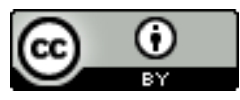

